\title{
Biossegurança aplicada à Odontologia na Universidade Federal do Pará, Cidade de Belém, Estado do Pará, Brasil
}

\author{
Biosafety applied to Dentistry at the Universidade Federal do Pará, in the City of Belém, Pará State, \\ Brazil
}

Bioseguridad aplicada a la Odontología en la Universidade Federal do Pará, Ciudad de Belém, Estado de Pará, Brasil

Diandra Costa Arantes

Programa de Pós-graduação em Odontologia, Universidade Federal do Pará, Belém, Pará, Brasil

Caio de Andrade Hage

Hospital Universitário João de Barros Barreto, Belém, Pará, Brasil

\author{
Liliane Silva do Nascimento \\ Faculdade de Odontologia, Universidade Federal do Pará, Belém, Pará, \\ Brasil
}

Flávia Sirotheau Correa Pontes

Faculdade de Odontologia, Universidade Federal do Pará, Belém, Pará, Brasil

\begin{abstract}
RESUMO
O presente estudo objetivou analisar o conhecimento e a aplicação das normas de biossegurança entre acadêmicos de Odontologia da Universidade Federal do Pará, a fim de verificar as divergências entre dois diferentes momentos da formação profissional e superar as deficiências detectadas. Trata-se de um estudo longitudinal, para o qual foi utilizado um questionário constituído por 17 perguntas objetivas, aplicado aos acadêmicos em dois momentos: após seu primeiro semestre de prática clínica e após a conclusão do curso. Foram pesquisados 35 discentes na primeira coleta e 30, na segunda. Ao final do curso, 100\% diziam-se bem informados sobre biossegurança; o processo de desinfecção mais aplicado ocorreu nas peças de mão; e o método de esterilização mais empregado foi a autoclave. No decorrer do curso, aumentou a frequência de uso de sobreluvas, porém os óculos especiais, os sapatos fechados e o propé foram os equipamentos de proteção individual mais negligenciados. Grande parte dos acadêmicos desconhecia o fluxo de atendimento para acidentes com pérfuro-cortantes, ainda que estes tenham ocorrido em $30 \%$ dos estudantes durante a graduação. São fundamentais a educação em biossegurança e a conscientização dos alunos quanto à adoção de hábitos corretos para o controle de infecção cruzada, desde a graduação e, futuramente, como cirurgiões-dentistas.
\end{abstract}

Palavras-chave: Estudantes de Odontologia; Exposição a Agentes Biológicos; Equipamentos de Proteção; Vacinação; Acidentes de Trabalho.

\section{INTRODUÇÃO}

A década de 1980, quando veio à tona o problema da aids e houve o primeiro relato de contaminação por acidente de trabalho em saúde, foi marcada pelo início das primeiras discussões e estudos acerca da biossegurança relacionada à Odontologia. Nessa década, também, surgiram publicações acerca do controle de infecção em Odontologia, o que alertou a comunidade de saúde sobre o perigo de transmissão ocupacional de agentes infecciosos, obrigou este setor a aperfeiçoar os métodos de

\footnotetext{
Correspondência / Correspondence / Correspondencia:

Diandra Costa Arantes

Universidade Federal do Pará, Faculdade de Odontologia

Rua Augusto Corrêa, $n^{\circ} 1$. Bairro: Guamá

CEP: 66075-110 Belém-Pará-Brasil

Tel.: +55 (91) 3201-6796

E-mail: arantesdiandra@yahoo.com.br
}

prevenção de risco de contaminação adotados pelos cirurgiões-dentistas e impulsionou maior preocupação com a biossegurança ${ }^{1,2}$.

Porém foi apenas em 2002 que o Ministério da Saúde constituiu a Comissão de Biossegurança em Saúde que, dentre suas atribuições, objetiva "participar e acompanhar, nos âmbitos nacional e internacional, da elaboração e da reformulação de normas de biossegurança" e, desde então, vários conceitos foram desenvolvidos e relacionados ao termo biossegurança ${ }^{3}$.

Esta pesquisa toma como base o conceito da Comissão Técnica de Biossegurança Fiocruz, que define: "biossegurança é o conjunto de ações voltadas para prevenção, minimização ou eliminação de riscos inerentes às atividades de pesquisa, produção, ensino, desenvolvimento tecnológico e prestação de serviços (...)"3.

O cirurgião-dentista está vulnerável ao risco de infecção transmitida por agentes infecciosos dentro do 
ambiente clínico por diferentes vias: por contato direto com lesões infecciosas, sangue e saliva contaminados; por contato indireto por meio de micro-organismos presentes em instrumentos, equipamentos e superfícies; por aspiração de micro-organismos nos aerossóis; e pelo contato entre pessoas ${ }^{1,4,5,6}$.

Logo, o controle da transmissão de doenças é um desafio para o profissional de Odontologia, já que ele atua sobretudo na cavidade oral, um ambiente com múltiplas espécies de microbiotas, de difícil acesso e de estreita relação com o rosto dos profissionais, devido à proximidade, na posição em que trabalham ${ }^{7,8}$.

As doenças passíveis de transmissão durante um tratamento odontológico abrangem desde a influenza até pneumonia, conjuntivite, herpes, tuberculose, hepatites $B$ e $C$, aids, sífilis, entre outras ${ }^{5,6}$.

Nesse contexto, objetivando evitar o contágio e manter o controle da saúde na equipe profissional e, também, em seus pacientes, a vacinação contra essas patologias antes do início da atividade clínica é uma das mais efetivas maneiras de prevenção, já que as medidas pós-exposição não são totalmente confiáveis e inúmeros são os instrumentos pérfuro-cortantes, empregados no cotidiano dessa atividade, que podem ocasionar acidentes?.

Segundo a Fundação Oswaldo Cruz, as vacinas recomendadas para os profissionais da saúde são: BCG, tríplice viral, hepatites $A$ e $B$, dupla bacteriana do tipo adulto, tríplice bacteriana do tipo adulto, varicela, influenza e antimeningocócica C conjugada ${ }^{3}$. ○ Ministério da Saúde disponibiliza algumas dessas vacinas gratuitamente em unidades de saúde, como é o caso da vacina contra hepatite $B$, que induz imunidade em 90 a $95 \%$ dos $\operatorname{casos}^{9,10}$

Contudo, não se podem esquecer as moléstias para as quais ainda não foi desenvolvida uma alternativa de imunoprofilaxia, como é o caso do HCV e do HIV ${ }^{10,11}$.

Diante disso, evitar as exposições ocupacionais torna-se a forma de prevenção primária para a transmissão de patógenos ${ }^{12}$. Doenças podem ser evitadas com medidas preventivas básicas, como o uso dos equipamentos de proteção individual (EPIs) e de técnicas de proteção coletiva que englobam a esterilização dos instrumentais, a desinfecção de superfícies, as barreiras para objetos (filmes plásticos e papéis de revestimento), a proteção do paciente e o gerenciamento de resíduos ${ }^{1,13,14}$.

Os EPls básicos para segurança e redução de riscos no atendimento odontológico são: gorro, luvas, sobreluvas, máscaras, jalecos, sapatos fechados e óculos de proteção ${ }^{6,7}$.

Cabe frisar ainda a relevância da constante informação e atualização dos profissionais como forma de controle da transmissão de patologias, visto que a educação é a melhor forma de prevenção².

Embora os acadêmicos dos cursos de Odontologia ainda não sejam profissionais, realizam atividades práticas nas clínicas de suas instituições de ensino e, igualmente, são expostos aos riscos inerentes à profissão ${ }^{15}$.
Nessa dimensão, esta pesquisa visou a analisar - conhecimento e a aplicação das normas de biossegurança por um mesmo grupo de alunos da Faculdade de Odontologia da Universidade Federal do Pará (UFPA) no início da prática clínica e após a conclusão do curso, a fim de verificar as divergências entre esses dois momentos da formação profissional e superar as deficiências detectadas.

\section{MATERIAIS E MÉTODOS}

Esta pesquisa foi aprovada pelo Comitê de Ética em Pesquisa em Seres Humanos do Instituto de Ciências da Saúde da UFPA, sob o número de protocolo 074/09.

Diferentemente de outros estudos que abordam a mesma temática, esta pesquisa analisou um mesmo grupo de alunos em dois momentos distintos do curso de graduação em Odontologia: o período referente ao início da prática clínica e o momento de conclusão do curso antes da formatura, a fim de verificar se as mesmas pessoas alteraram seu comportamento em relação ao cumprimento de normas de biossegurança no decorrer da prática e da construção do conhecimento em atendimento clínico.

É um estudo longitudinal, cuja amostra pareada inclui todos os alunos do curso de Odontologia da UFPA que cursavam o quinto período no primeiro semestre de 2009 (dos turnos manhã e tarde), somando 35 alunos. Foi uma amostra sem reposição.

Anteriormente à coleta de dados, o instrumento foi elaborado pelos autores especialmente para essa pesquisa e organizado em forma de questionário, com 17 perguntas objetivas, elaboradas com base no manual de biossegurança da instituição de ensino e na literatura anteriormente revisada sobre o assunto.

questionário foi previamente testado com cinco alunos de nível de conhecimento aproximado, apenas para verificação de possível falta de clareza nas perguntas. Os temas abordados foram: conhecimento sobre biossegurança; percepção em relação ao risco de contaminação na clínica universitária; esterilização e desinfecção; uso dos EPls; vacinação; destino de pérfuro-cortantes; e influência do número de pacientes a serem atendidos em um mesmo turno em relação ao não cumprimento das normas de biossegurança.

Os alunos foram convidados a participar da pesquisa e assinaram um Termo de Consentimento Livre e Esclarecido. $\bigcirc$ questionário foi aplicado individualmente pelo pesquisador em horário adequado a não prejudicar as atividades da universidade.

$\bigcirc$ questionário utilizado foi igual nos dois momentos de pesquisa: primeira coleta de dados no início da prática clínica (quinto período, em junho de 2009) e, na segunda coleta de dados, após a conclusão do curso (décimo período, em dezembro de 2011 ).

Os dados obtidos foram tabulados e analisados por estatística descritiva e os grupos foram comparados por meio de estatística não paramétrica pelo Teste de McNemar, para dados nominais, e pelo Teste de Wilcoxon, para dados ordinais. As diferenças foram consideradas estatisticamente significativas para $p<0,05$. 


\section{RESULTADOS}

O questionário foi respondido por 35 discentes na primeira coleta de dados, sendo 19 do sexo masculino e 16 do sexo feminino. Em relação à segunda coleta, 30 acadêmicos (16 do sexo masculino e 14 do sexo feminino) participaram da pesquisa, pois no intervalo entre as coletas, que foi de dois anos e meio de curso, três foram reprovados, um trancou a matrícula e um não foi encontrado no período das entrevistas.

No que diz respeito à frequência de uso de EPls (Tabela 1), houve diferença estatisticamente significante para $\bigcirc$ uso de óculos especiais $(p=0,0431)$ e propé $(p=0,0111)$ entre dois distintos momentos de avaliação (Figura 1).
Início da clínica 0\%

$33,4 \%$

$3,3 \%$

$57,2 \%$

Final do curso

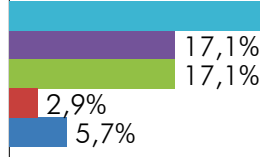

$17,1 \%$

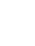

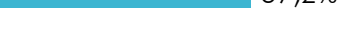

Todos

Todos, exceto propé

Todos, exceto óculos

Todos, exceto óculos e propré

Outro

Figura 1 - Distribuição dos alunos quanto ao uso silmutâneo de EPls durante atendimento odontológico. Belém, $2009 / 2011$

Tabela 1 - Frequência do uso de EPIs por acadêmicos de Odontologia durante atendimento de pacientes. Belém, 2009/2011

\begin{tabular}{|c|c|c|c|c|}
\hline \multirow{3}{*}{ EPI } & \multicolumn{4}{|c|}{ Período } \\
\hline & \multicolumn{2}{|c|}{ Início da clínica } & \multicolumn{2}{|c|}{ Final do curso } \\
\hline & $\mathrm{Fa}$ & $\mathrm{Fr}$ & $\mathrm{Fa}$ & $\mathrm{Fr}$ \\
\hline \multicolumn{5}{|l|}{ Jaleco } \\
\hline Sempre & 35 & 100 & 30 & 100 \\
\hline Às vezes & - & - & - & - \\
\hline Nunca & - & - & - & - \\
\hline \multicolumn{5}{|c|}{ Sapato fechado } \\
\hline Sempre & 30 & 85,7 & 20 & 66,7 \\
\hline Às vezes & 5 & 14,3 & 10 & 33,3 \\
\hline Nunca & - & - & - & - \\
\hline \multicolumn{5}{|l|}{ Luva } \\
\hline Sempre & 35 & 100 & 30 & 100 \\
\hline Às vezes & - & - & - & - \\
\hline Nunca & - & - & - & - \\
\hline \multicolumn{5}{|c|}{ Óculos especiais } \\
\hline Sempre & 18 & 51,4 & 18 & 60 \\
\hline Às vezes & 16 & 45,7 & 12 & 40 \\
\hline Nunca & 1 & 2,9 & - & - \\
\hline \multicolumn{5}{|l|}{ Gorro } \\
\hline Sempre & 34 & 97,1 & 27 & 90 \\
\hline Às vezes & 1 & 2,9 & 3 & 10 \\
\hline Nunca & - & - & - & - \\
\hline \multicolumn{5}{|l|}{ Propé } \\
\hline Sempre & 18 & 51,4 & - & - \\
\hline Às vezes & 14 & 40 & 13 & 43,3 \\
\hline Nunca & 3 & 8,6 & 17 & 56,7 \\
\hline \multicolumn{5}{|l|}{ Sobreluvas } \\
\hline Sempre & 1 & 2,9 & 1 & 3,3 \\
\hline Às vezes & 27 & 77,1 & 18 & 60 \\
\hline Nunca & 7 & 20 & 11 & 36,7 \\
\hline \multicolumn{5}{|l|}{ Máscara } \\
\hline Sempre & 34 & 97,1 & 30 & 100 \\
\hline Às vezes & 1 & 2,9 & - & - \\
\hline Nunca & - & - & - & - \\
\hline
\end{tabular}

Fa: frequência absoluta; Fr: frequência relativa em relação ao total de pesquisados (\%).

Sinal convencional utilizado: - Dado numérico igual a zero não resultante de arredondamento. 
Acerca do risco de contaminação cruzada na clínica universitária (Tabela 2), não houve diferença estatisticamente significante da percepção dos alunos quanto ao assunto $(p=0,5755)$.
Já com relação à esterilização de instrumentais (Tabela 2), o valor de $\mathrm{p}=0,0180$ indica que há distinção de hábitos entre o início da clínica e o final do curso.

Tabela 2 - Dados obtidos pelos questionários. Belém, 2009/2011

\begin{tabular}{|c|c|c|c|c|}
\hline \multirow{3}{*}{ Perguntas e respostas do questionário } & \multicolumn{4}{|c|}{ Período } \\
\hline & \multicolumn{2}{|c|}{ Início da clínica } & \multicolumn{2}{|c|}{ Final do curso } \\
\hline & $\mathrm{Fa}$ & $\mathrm{Fr}$ & $\mathrm{Fa}$ & $\mathrm{Fr}$ \\
\hline \multicolumn{5}{|l|}{ Considera-se bem informado sobre biossegurança? } \\
\hline $\operatorname{Sim}$ & 31 & 88,6 & 30 & 100 \\
\hline Não & - & - & - & - \\
\hline Um pouco & 4 & 11,4 & - & - \\
\hline \multicolumn{5}{|l|}{ Qual o risco de contaminação cruzada na clínica? } \\
\hline Nenhum & - & - & - & - \\
\hline Grande & 10 & 28,6 & 15 & 50 \\
\hline Médio & 17 & 48,6 & 12 & 40 \\
\hline Pequeno & 8 & 22,8 & 3 & 10 \\
\hline \multicolumn{5}{|l|}{ Qual a frequência de esterilização dos instrumentais? } \\
\hline Sempre & 28 & 80 & 30 & 100 \\
\hline Às vezes & - & - & - & - \\
\hline Nunca & 7 & 20 & - & - \\
\hline \multicolumn{5}{|l|}{ Qual o método de esterilização utilizado? } \\
\hline Autoclave & 31 & 88,6 & 27 & 90 \\
\hline Químico & 1 & 2,8 & - & - \\
\hline Estufa & - & - & - & - \\
\hline Autoclave e químico & 3 & 8,6 & 3 & 10 \\
\hline \multicolumn{5}{|l|}{ O que é desinfetado sempre antes de cada paciente? } \\
\hline Peças de mão & 30 & 85,7 & 30 & 100 \\
\hline Cadeira odontológica & 29 & 82,9 & 23 & 76,7 \\
\hline Bancada & 26 & 74,3 & 18 & 60 \\
\hline Nenhum & - & - & - & - \\
\hline \multicolumn{5}{|l|}{ Retira todos os EPls antes de sair da clínica? } \\
\hline Sim & 23 & 65,7 & 18 & 60 \\
\hline Não & 12 & 34,3 & 12 & 40 \\
\hline \multicolumn{5}{|l|}{ Quando são usadas as sobreluvas de plástico? } \\
\hline Atender ao telefone & 25 & 71,4 & 21 & 70 \\
\hline Tomadas radiográficas & 17 & 48,6 & 20 & 66,7 \\
\hline Pegar materiais fora da bancada & 12 & 43,3 & 13 & 43,3 \\
\hline Abrir portas & 18 & 51,4 & 18 & 60 \\
\hline Outros & 14 & 40 & 8 & 26,7 \\
\hline \multicolumn{5}{|l|}{ Conhece campanhas de vacinação realizadas pela UFPA? } \\
\hline Sim & 15 & 42,9 & 10 & 33,3 \\
\hline Não & 20 & 57,1 & 20 & 66,7 \\
\hline \multicolumn{5}{|l|}{ Conhece o fluxo para acidentes com pérfuro-cortantes? } \\
\hline Sim & 13 & 37,1 & 12 & 40 \\
\hline Não & 22 & 62,9 & 18 & 60 \\
\hline \multicolumn{5}{|l|}{ Já sofreu acidente com pérfuro-cortantes? } \\
\hline $\operatorname{Sim}$ & 8 & 22,9 & 9 & 30 \\
\hline Não & 27 & 77,1 & 21 & 70 \\
\hline \multicolumn{5}{|c|}{$\begin{array}{l}\text { O número de pacientes influencia no cumprimento de normas de } \\
\text { biossegurança? }\end{array}$} \\
\hline Sim & 9 & 25,7 & 10 & 33,3 \\
\hline Não & 26 & 74,3 & 20 & 66,7 \\
\hline
\end{tabular}

Fa: frequência absoluta; Fr: frequência relativa em relação ao total de pesquisados (\%).

Sinal convencional utilizado: - Dado numérico igual a zero não resultante de arredondamento. 
Além disso, pelo teste de McNemar não houve diferença estatisticamente significante $(p=0,7539)$ sobre a opinião quanto à influência do número de pacientes a serem atendidos em relação ao cumprimento de normas de biossegurança (Tabela 2).

\section{DISCUSSÃO}

Com essa pesquisa verificou-se que não houve o cumprimento dito ideal das normas de biossegurança - preconizadas tanto pelo manual de biossegurança da UFPA, como pelo Ministério da Saúde (MS) - em nenhuma das duas fases de coleta de dados.

O risco de infecção cruzada dentro do ambiente odontológico ocorre tanto pelo contato com material orgânico quanto durante procedimentos que geram aerossol ou exigem manipulação ${ }^{14,16}$. Neste estudo verificou-se que os acadêmicos tinham o conhecimento de que esse risco está presente em sua atividade clínica diariamente, bem como acreditavam que o mesmo cresceu até o final do curso, possivelmente devido aos procedimentos de maior grau de dificuldade que realizaram e ao maior fluxo de pacientes atendidos no decorrer dos semestres.

Todavia, apesar da noção do risco de infecção cruzada a que estão expostos, alguns acadêmicos negligenciavam procedimentos básicos de prevenção, como a desinfecção, processo no qual é eliminada a maioria dos microrganismos patogênicos presentes em objetos inanimados, já que o nível de contaminação dos mesmos não depende exclusivamente do número de pacientes e operadores, mas também da falha do uso de filmes plásticos e da limpeza com agentes químicos apropriados ${ }^{14,17}$. Esta medida não foi aplicada nas peças de mão por alguns alunos no primeiro momento da pesquisa, mas, felizmente, por todos eles, no final do curso. Entretanto, diminuiu o número dos que desinfetavam a bancada e a cadeira odontológica, apesar de este procedimento estar previsto no manual de biossegurança da instituição, o qual preconiza que "equipo e bancadas devem passar por desinfecção e colocação de barreiras previamente a cada atendimento"18. Estes índices deixam a desejar quando comparados com uma pesquisa realizada com profissionais no Estado de São Paulo, pois todos os cirurgiões-dentistas afirmaram utilizar álcool 70\% nas bancadas e apenas aproximadamente 3\% não realizava qualquer tipo de desinfecção na cadeira odontológica $^{13}$.

Ainda como método para evitar a infecção cruzada, vários estudos abordaram 0 uso de sobreluvas. Garbin et $a^{13}$ identificaram que $37 \%$ dos profissionais pesquisados as usavam para procedimentos não operatórios, como abrir gavetas ou atender ao telefone, e Pimentel et $\mathrm{al}^{16}$ constataram em sua pesquisa com acadêmicos que 29,1\% deles evitavam tocar objetos fora da bandeja estéril após calçar as luvas de látex e/ou não as trocavam para continuar o atendimento.

Nesta pesquisa, na primeira coleta, menos da metade dos acadêmicos revelaram calçar sobreluvas de plástico para executar tomadas radiográficas, entretanto na segunda, esse número felizmente aumentou, bem como cresceu a porcentagem dos que as utilizavam para pegar outros materiais, mas ainda assim representa uma porção pequena, pois o manual de biossegurança já citado frisa que "para segurar embalagens, manipular ampola de R-X etc, deve-se descartar a luva ou colocar sobreluva"18.

Além disso, o MS instrui os profissionais a não manipularem os objetos que não sejam do campo de trabalho, como caneta e prontuário, enquanto estiverem de luvas e, com elas, nunca atender ao telefone, abrir portas ou gavetas, devendo-se optar por usar uma sobreluva ${ }^{5}$.

Em relação ao uso de EPls, os óculos de proteção foram um dos equipamentos menos utilizados. Apesar da frequência de uso tipificada como "sempre" ter subido, o uso dos mesmos "às vezes" é preocupante porque gotículas infectadas podem atingir e lesar a mucosa ocular e a córnea do dentista se não houver uma barreira entre a boca do paciente e os olhos do profissional ${ }^{12}$. Porém esse desleixo é um fato que se constatou em diversos estudos, os quais demonstraram que uma média de $28 \%$ dos acadêmicos não os utilizavam, em algumas universidades ${ }^{16,19,20}$.

Quanto aos demais EPls, como luvas, gorro, máscara e jaleco, outros autores obtiveram valores semelhantes aos encontrados nesta investigação ${ }^{13,16,20,21}$. Porém, uma falha desta pesquisa foi a de não verificar se o uso dos equipamentos é feito de forma correta, pois Vasconcelos et $a^{21}$, em um estudo observacional, verificaram o uso inadequado desses equipamentos, em grande parcela para o gorro, mas, também, em relação à máscara e à luva de procedimento.

Ainda nesse contexto, os discentes também foram questionados quanto à remoção de todos esses equipamentos para sair da clínica universitária. Menos da metade (na segunda coleta) deles afirmou não os retirar, porção esta inferior a outro estudo nordestino, no qual cerca de dois terços dos alunos declararam transitar pelos corredores de jaleco ${ }^{20}$.

A esterilização de instrumentais odontológicos é vital para a redução da infecção cruzada, uma vez que permite a eliminação de praticamente todos os microrganismos neles presentes ${ }^{17}$. No início da atividade clínica, 20\% dos pesquisados revelaram esterilizar seus instrumentais apenas às vezes, o que era bastante preocupante, mas, provavelmente, justificado pela utilização de alguns instrumentais 
apenas em laboratórios. Felizmente, no encerramento das atividades clínicas, todos afirmaram sempre esterilizá-los.

Em outra universidade do País, registraram-se casos em que os materiais não foram esterilizados ou desinfectados - destacando-se os posicionadores radiográficos, bandejas metálicas, gaze e escovas de Robinson - e aproximadamente 4\% dos cirurgiões-dentistas pesquisados por autores estrangeiros declararam não esterilizar todos os seus instrumentais, o que é um dado de alerta ${ }^{21,22}$.

Quanto ao método de esterilização, a autoclave foi a mais empregada pelos discentes, provavelmente porque este é o único método oferecido por sua instituição de ensino. De maneira similar, uma pesquisa científica realizada em diversas universidades públicas do Brasil constatou que $81,2 \%$ das mesmas têm disponíveis a autoclave e a estufa; apenas 6,2\% fornecem apenas a autoclave; e 12,5\% combinam a autoclave com um método químico ${ }^{17}$. Nesse sentido, os dados encontrados com os acadêmicos da UFPA são satisfatórios, pois as autoclaves oferecem boa segurança biológica. Estudos de Correa et $\mathrm{al}^{23}$ obtiveram somente $8,3 \%$ de insucesso desse método, verificado com indicadores químicos internos e biológicos, em comparação aos 21,5\% de insucesso na esterilização com estufas, índice esse que pode ser considerado alto, quando se trata de biossegurança.

Um número estimado de 400 milhões de pessoas, em todo o mundo, estão ativamente infectadas por hepatite $\mathrm{B}^{24}$. Diante disso, ainda é insatisfatório que, ao final do curso, nem todos os alunos estejam imunizados contra essa patologia. Nessa dimensão, outros autores observaram o índice para estudantes de Odontologia de diversas regiões do Brasil e o mesmo variou de $38 \%$ a $99 \%$ de vacinados, como também variou o índice para dentistas de 74\% a $100 \% 9,11,14,19,20,22,25$.

Além disso, mesmo ao final do curso, cerca de dois terços dos futuros profissionais desconheciam as campanhas de vacinação promovidas pela UFPA, que ocorreram nos anos de 2008, 2009 e 2010. Desta forma, importa aumentar a divulgação das referidas campanhas pela instituição, inclusive por meio de professores dos centros de ciências da saúde $\mathrm{e}^{26}$.

A imunização contra patologias para as quais vacinas já foram desenvolvidas é fundamental devido ao constante risco de acidentes com instrumentos pérfuro-cortantes, principalmente os alunos de graduação, devido à inexperiência e ao variável nível de habilidade técnica ${ }^{27}$.
As pesquisas que abordam esse tema o fazem geralmente de duas maneiras: 1) detectando o número de acadêmicos e profissionais que já foram vítimas de acidentes com pérfuro-cortantes dentro de cada categoria; estes dados variam de $9 \%$ a $59 \%$ para discentes e de $38 \%$ a $81 \%$ para cirurgiões-dentistas; 2) verificando, dentro de uma instituição de ensino, o quão representativos são tais acidentes em relação a todos os indivíduos que nela circulam (alunos, professores, funcionários e pacientes); os resultados obtidos mostram que de $76 \%$ a $98 \%$ das vítimas eram acadêmicos $8,12,25,27,28,29$.

valor obtido nesta pesquisa, de que aproximadamente um terço dos discentes finalizaram - curso já tendo sofrido pelo menos um acidente desta natureza, é um fator de alerta, apesar de estar dentro da média quando comparado com os dados acima. Um estudo internacional, cujos dados revelaram que aproximadamente $23 \%$ dos dentistas deste País se acidentaram pelo menos uma vez por semana, mostrou-se surpreendente devido ao risco de morbidade e até mesmo mortalidade diretamente relacionados ao acidente ${ }^{22}$.

decepcionante, porém, é que mesmo todos os acadêmicos considerando-se bem informados sobre biossegurança na última investigação, menos da metade deles conhecia o fluxo de atendimento para acidentes com pérfuro-cortantes, incluindo a unidade de referência de Belém para tais casos (URE DIP-SESPA), onde há testes rápidos para HIV e profissionais capacitados para trabalhar com essas situações.

Apesar da amostra representar o objetivo de estudo proposto pelos autores, pesquisas com maior número de indivíduos fazem-se interessantes a fim de ratificar a prática errônea e verificar se ela ocorre por falta real de conhecimento de normas ou se, na verdade, por descaso com relação a normas cediças, já que educação em biossegurança e, primordialmente, a conscientização dos alunos quanto à relevância da mesma para a saúde pessoal são fundamentais para que os mesmos criem hábitos corretos no que tange ao controle de infecção cruzada desde a graduação e, futuramente, como profissionais.

\section{CONCLUSÃO}

Embora boa parte dos acadêmicos pesquisados afirme ter bons conhecimentos em biossegurança e tenha noção do risco de infecção cruzada dentro de sua clínica universitária, muitos deles não aplicam todas as normas de biossegurança previstas pela Agência Nacional de Vigilância Sanitária (ANVISA), pelo MS e pelo manual de biossegurança da instituição. 


\title{
Biosafety applied to Dentistry at the Universidade Federal do Pará, in the City of Belém, Pará State, Brazil
}

\begin{abstract}
This study aimed to analyze the knowledge and application of biosafety standards among Dentistry students at the Universidade Federal do Pará, in order to verify differences between two different moments of professional formation and correct deficiencies. This is a longitudinal study, in which a questionnaire was used, consisting of 17 objective questions, applied to the students in two moments: after their first semester of clinical practice and after the course conclusion. In the first sample, 35 students were surveyed and 30 in the second. By the end of the course, $100 \%$ of the students said they were well informed about biosafety; the most applied disinfection process occurred in handpieces; and the most used sterilization method was the autoclave; during the course, the use of double gloves increased, however the most neglected personal protective equipment were special glasses, enclosed shoes and shoe cover. Most of the students were unaware of the flow of care for needlestick accidents, although 30\% of them have had this experience during the graduation course. Biosafety education and students awareness are fundamental regarding the adoption of correct habits to control the cross-infection since graduation and, eventually, as dentists.
\end{abstract}

Keywords: Students, Dental; Exposure to Biological Agents; Protective Devices; Vaccination; Accidents, Occupational.

\section{Bioseguridad aplicada a la Odontología en la Universidade Federal do Pará, Ciudad de Belém, Estado de Pará, Brasil}

\section{RESUMEN}

El presente estudio tuvo como objetivo analizar el conocimiento y la aplicación de las normas de bioseguridad entre académicos de Odontología de la Universidade Federal do Pará, con el fin de comprobar las divergencias entre dos diferentes momentos de la formación profesional y superar las deficiencias detectadas. Se trata de un estudio longitudinal, para lo que se utilizó un cuestionario, constituido por 17 preguntas objetivas, aplicado a los académicos en dos momentos: después de su primer semestre de práctica clínica y después de la conclusión del curso. Fueron analizados 35 discentes en la primera colecta y 30, en la segunda. Al final del curso, 100\% se decía bien informado sobre bioseguridad; el proceso de desinfección más aplicado ocurrió en las piezas de mano; y el método de esterilización más empleado fue la autoclave. Durante el curso, aumentó la frecuencia del uso de sobreguantes, pero los lentes especiales, los zapatos cerrados y las zapatillas desechables (propé) fueron los equipamientos de protección individual más descuidados. Gran parte de los académicos desconocía el flujo de atención para accidentes con perfuro cortantes, aunque estos hayan ocurrido en 30\% de los estudiantes durante la graduación. Es fundamental la educación en bioseguridad y la concienciación de los alumnos con relación a la adopción de hábitos correctos para el control de infección cruzada, desde la graduación y, en el futuro, como cirujanos dentistas.

Palabras clave: Estudiantes de Odontología; Exposición a Agentes Biológicos; Equipos de Seguridad; Vacunación; Accidentes de Trabajo.

\section{REFERÊNCIAS}

1 Rodrigues MP, Domingos Sobrinho M, Silva EM. Os cirurgiões dentistas e as representações sociais da AIDS. Cienc Saude Coletiva. 2005 abr-jun;10(2):463-72.

2 Andrade AC, Sanna MC. Ensino de biossegurança na graduação em enfermagem: uma revisão da literatura. Rev Bras Enferm. 2007 setout;60(5):569-72.

3 Comissão de Biossegurança da Fundação Oswaldo Cruz. Portaria 131/2003 [Internet]. 2003 [citado 2012 jun 10]. Disponível em: http://www.fiocruz.br/ biosseguranca/Bis/StartBIS.htm.
4 Bulgarelli AF, Torquato TM, Costa LSS, Ferreira ZA. Avaliação das medidas de biossegurança no controle de infecção cruzada durante tratamento periodontal básico. Rev Bras Odontol. 2001 mai-jun;58(3): 188-90.

5 Ministério da Saúde (BR). Controle de infecções e a prática odontológica em tempos de AIDS: manual de condutas. Brasília: Ministério da Saúde; 2000.

6 Rodrigues ISC, Ribeiro IRA, Araújo VM, Lima DLF. Acidentes ocupacionais na graduação em Odontologia: retrospectiva de 10 anos. Rev Bras Promoç Saude. 2009 out-dez;22(4):240-4. 
7 Serratine ACP, Pacheco E, Miero M. Avaliação da integridade das luvas cirúrgicas após a utilização em cirurgias odontológicas. Arq Catarin Med. 2007;36(1):85-9.

8 Bragança DPP, Fernandes MM, Sassi C, Francesquini Júnior L, Daruge Júnior E. Condutas do cirurgião-dentista frente a acidentes biológicos. Odonto. 2010 jan-jun; 18(35):37-44.

9 Cavalcanti FM, Melo RGSV, Patrício DPS, Zimmermann RD. Hepatite B: conhecimento e vacinação entre os acadêmicos da Faculdade de Odontologia de Caruaru-PE. Odontol Clin-Cient. 2009 jan-mar;8(1):59-65.

10 Ministério da Saúde (BR). Secretaria de Vigilância em Saúde. Departamento de Vigilância Epidemiológica. Hepatites virais: o Brasil está atento. 3. ed. Brasília: Ministério da Saúde; 2008.

11 Veloso HHP, Queiroga AS, Silveira RCJ, Canuto MR, Pontual AA. Avaliação de traumas físicos sofridos por acadêmicos. Rev Odontol Bras Central. $2011 ; 20(53): 151-4$

12 Brozoski MA, Traine AA, Naclério-Homem MG, Deboni MCZ. Ocorrência de acidentes pérfurocortantes em um curso de odontologia. RGO. 2010 jan-mar;58(1):77-80.

13 Garbin CAS, Moimaz SAS, Almeida MEL, Ferreira NF. A importância da biossegurança para o cirurgião-dentista. J Bras Clin Odontol Integr. 2004;8(45):216-21.

14 Pinelli C, Garcia PPNS, Campos JADB. Biossegurança e odontologia: crenças e atitudes de graduandos sobre o controle da infecção cruzada. Saude Soc. 2011 abr-jun;20(2):448-61.

15 Canalli RTC, Moriya TM, Hayashida M. Prevenção de acidentes com material biológico entre estudantes de enfermagem. Rev Enferm UERJ. 2011 jan-mar; 19(1):100-6.

16 Pimentel MJ, Batista Filho MMV, Rosa MRD, Santos JP. Utilização dos equipamentos de proteção individual pelos acadêmicos de odontologia no controle da infecção cruzada. Rev Bras Odontol. 2009 jul-dez;66(2):21 1-5.

17 Sasamoto SAA, Tipple AFV, Souza ACS, Paiva EMM, Souza CP, Pimenta FC. Evaluation of central supply units in public dental medicine colleges in Brazil. Braz J Infect Dis. 2004 Dec;8(6):445-53.

18 Universidade Federal do Pará. Faculdade de Odontologia. Comissão de Biossegurança. Manual de Biossegurança da Faculdade de Odontologia da UFPA. 2. ed. rev. Belém: Universidade Federal do Pará; 2009.
19 Lima AA, Azevedo AC, Fonseca AGL, Silva JLM, Padilha WWN. Acidentes ocupacionais: conhecimento, atitudes e experiências de estudantes de odontologia da Universidade Federal da Paraíba. Pesq Bras Odontoped Clin Integr. 2008 setdez;8(3):327-32.

20 Granville-Garcia AF, Batista BB, Cavalcanti AL, D'Avila S, Lins RDAU, Menezes VA. Adesão e conhecimento de medidas de proteção individual contra a Hepatite B entre estudantes de Odontologia. Odontol Clin-Cientific. 2009 outdez;8(4):325-30.

21 Vasconcelos MMVB, Brasi CMV, Mota CCBO, Carvalho NR. Avaliação das normas de biossegurança nas clínicas odontológicas da UFPE. Odontol Clin-Cient. 2009 abr-jun;8(2):151-6.

22 Cheng H-C, Su C-Y, Yen AM-F, Huan C-F. Factors affecting occupational exposure to needlestick and sharps injuries among dentists in Taiwan: a nationwide survey. PLoS One. 2012 Apr;7(4):e34911.

23 Corrêa EG, Castilho ARF, Pereira CV. Indicadores químicos e biológicos da eficácia de esterilização por autoclave ou estufa. Rev Odonto Cienc. 2009 abr-jun;24(2): 156-60.

24 Resende VLS, Abreu MHG, Paiva SM, Teixeira $R$, Pordeus IA. Concerns regarding hepatitis $B$ vaccination and post-vaccination test among Brazilian dentists. Virol J. 2010 Jul;7(154): 1-9.

25 Stewardson DA, Palenik CJ, McHugh ES, Burke FJT. Occupational exposures occurring in students in a UK dental school. Eur J Dent Educ. 2002 Aug;6(3):104-13.

26 Coordenadoria de Vigilância à Saúde e Segurança do Trabalhador [Internet]. 2012 [citado 2012 set 1]. Disponível em: http://www.portal.ufpa.br/imprensa/ todasNoticias. php.

27 Machado-Carvalhais HP, Ramos-Jorge ML, Auad SM, Martins LHPM, Paiva SM, Pordeus IA. Occupational exposure to potentially infectious biological material in a dental teaching environment. J Dent Educ. 2008 Oct;72(10): 1201-8.

28 Veloso HHP, Queiroga AS, Silveira RCJ, Canuto MR, Pontual AA. Avaliação de traumas físicos sofridos por acadêmicos. Rev Odontol Bras Central. $2011 ; 20(53): 15-4$.

29 Nascimento LS, Assunção LRS, Silva Júnior NG, Pedreira EN, Silva RLC. Acidentes com pérfuro-cortantes na Faculdade de Odontologia da UFPA: visualização de um Cenário. Rev Odontol Bras Central. 2012;21 (57):462-7. 